\title{
Influência das energias de superfícies e interfaces na densificação durante a sinterização - um modelo geométrico
}

\author{
(The influence of surface and interface energies \\ during sintering - a geometric approach)
}

\author{
D. Gouvêa \\ Laboratório de Processos Cerâmicos, Departamento de Engenharia Metalúrgica e de Materiais \\ Escola Politécnica, Universidade de S. Paulo \\ Av. Prof. Mello Moraes 2463, Cidade Universitária, S. Paulo, SP, 05508-900 \\ dgouvea@usp.br
}

\begin{abstract}
Resumo
Devido a grande importância dada aos processos difusionais na sinterização, ou seja, a fatores cinéticos, pouco tem evoluído o conhecimento da sinterização no estado sólido do ponto de vista da energia do sistema de partículas. Alguns pesquisadores têm se esforçado em compreender a importância das energias da superfície dos poros e da interface do contorno de grão no processo de densificação e sua relação com os processos difusionais e a termodinâmica da sinterização. Contudo, pouco avanço tem sido obtido nesse sentido. O presente trabalho foi desenvolvido sobre um modelo geométrico simples de empacotamento de esferas sobre o qual foram realizados cálculos para se avaliar as variações de densidade, área específica dos poros e dos contornos de grão durante três situações: densificação sem crescimento de grãos, crescimento de grãos sem densificação e crescimento de grãos e densificação. Os resultados sugerem existir uma relação entre a evolução da microestrutura para a densificação ou não de um sistema de partículas em função da razão entre as energias do contorno de grão e da superfície dos poros.
\end{abstract}

Palavras-chave: sinterização, modelo, energia de superfície, contornos de grão.

\begin{abstract}
Sintering is a diffusional process and the mechanism of mass transport is assumed to control the phenomenon. Minor attention has been paid on the influence of surface and interface energies during sintering. Even if some works have tried to understand the influence of the modifications of the pore and grain boundary surfaces in the densification during sintering and their relationships with the difusional process, modest progresses have been reached. This work was developed from a simple model of packing spheres, allowing for calculating simultaneously the variation of pore, grain boundary and density for three situations: densification without grain growth, grain growth without densification and grain growth with densification. The results suggest a relationship between the microestrutural evolution during sintering with or without densification and the ratio of the surface energies of the grain boundary and the pores.
\end{abstract}

Keywords: sintering, surface energy, grain boundary, sintering model.

\section{INTRODUÇÃ̃O}

Estudos sobre a sinterização no estado sólido vêm sendo realizados nas últimas sete décadas na tentativa de se elaborar um modelo que, levando em consideração variáveis como: temperatura, composição química, distribuição de tamanho de grãos, distribuição de tamanho de poros e atmosfera, possa prever o comportamento da densificação em função do tempo de sinterização [1]. Contudo, atualmente não existe um modelo único que permita prever o comportamento de um sistema em suas diferentes etapas de eliminação de porosidade (inicial, intermediária e final), bem como a ausência de densificação observada em alguns sistemas, de forma satisfatória.

A força motriz para a sinterização de partículas, com ou sem densificação, é a eliminação do excesso de energia livre das superfícies e interfaces. A energia da superfície (poros) é considerada muito elevada em comparação à energia das interfaces (contornos de grãos) e desta forma, quando um sistema de partículas é submetido a um tratamento térmico onde os átomos apresentam certa mobilidade, deve ocorrer a eliminação dos poros e formação de contornos de grão, ou seja, densificação. Essa consideração é bastante aceitável quando o material a ser sinterizado é um metal. As ausências de direção preferencial de ligação (ligações covalentes) e a presença de cargas elétricas (ligações iônicas) desse tipo de material faz com que o átomo da superfície apresente um estado energético bastante desfavorável, pois por um lado a sua coordenação de equilíbrio não está satisfeita, por outro, o átomo no contorno de grão se apresenta em uma situação energética bastante mais favorável, já que sua coordenação pode 
estar muito próxima daquela de equilíbrio. Ao mesmo tempo, não é necessário que exista uma direção preferencial de ligação para o átomo presente no contorno de grão. O mesmo não pode ser considerado para materiais cerâmicos iônicos e covalentes. A presença de cargas nos materiais iônicos e de uma direção preferencial de ligação em materiais covalentes pode fazer com que a energia do contorno de grão se torne bastante elevada. A adsorção de gases e a segregação de íons aditivos ou contaminantes na superfície dos poros podem reduzir a sua energia [2] fazendo com que a diferença entre a energia do contorno de grãos e a dos poros se torne pequena. Levando em conta que estes fatores podem ocorrer simultaneamente, o motor da densificação pode ser prejudicado e a densificação pode não ocorrer em condições habituais de sinterização, sendo necessários recursos mais energéticos para a densificação do sistema como altas temperaturas, uso de pressão superior à atmosférica, controle de atmosfera entre outros. A importância da energia do contorno de grão nos modelos de sinterização é pequena sendo que existem poucos modelos que levam a sua contribuição em consideração.

A eliminação das superfícies e formação das interfaces esta ligada tanto a fatores termodinâmicos como a cinéticos destas e muito pouca atenção tem sido dada a estes fatores, principalmente devido à dificuldade de se avaliar essas grandezas durante o processo de sinterização. Outro fenômeno importante que deve ser considerado é que o crescimento dos grãos (coalescência) também está diretamente relacionado à modificação de superfícies e interfaces.

Este trabalho tem como objetivo calcular as variações de grandezas geométricas como tamanho de grãos, distância entre grãos, área do contorno de grãos, área do poro e volume de poro durante a sinterização de um sistema ideal de partículas nas diferentes condições, a saber: densificação sem crescimento de grãos, coalescência sem densificação e coalescência com densificação. É proposto um modelo geométrico de empacotamento de grãos em um sistema cúbico simples e os resultados são discutidos com relação à termodinâmica das superfícies e interfaces.

\section{MODELO GEOMÉTRICO}

\section{Definições}

Para simplificação do problema consideraremos um empacotamento cúbico simples de grãos esféricos e de raios idênticos. A célula primitiva bem como algumas variáreis a serem utilizadas no modelo geométrico como: o raio do grão (R), a metade da menor distância entre dois grãos (ou da distância do centro do grão ao contorno de grão $(\mathrm{H})$, o ângulo, $\theta$, entre R e H são mostrados na Fig. 1.

Outra grandeza apresentada que será utilizada posteriormente na discussão é o ângulo diedro, $\Psi$ (Fig. 1).

Com as variáveis definidas é possível o cálculo de algumas grandezas para a célula unitária como a área do contorno de grão $\left(\mathrm{A}_{\mathrm{CG}}\right)$, a área do poro $\left(\mathrm{A}_{\mathrm{p}}\right)$, o volume do grão $\left(\mathrm{V}_{\mathrm{G}}\right)$ e o volume do poro $\left(\mathrm{V}_{\mathrm{p}}\right)$ lembrando-se que existe um poro e um grão por célula unitária:

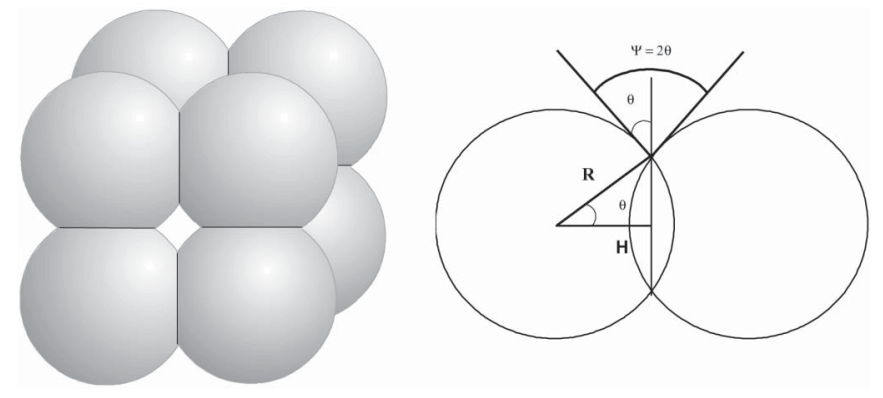

Figura 1: Modelo geométrico e algumas variáveis $(\mathrm{R}=$ raio da partícula, $\mathrm{H}=$ distância do centro da partícula ao contorno de grão, $\theta$ a ângulo entre $\mathrm{R} \mathrm{e} \mathrm{H} \mathrm{e} \Psi$ = ângulo diedro).

[Figure 1: Geometric model and some variables $(R=$ particle radius, $H=$ distance between the particle center and the grain boundary, $\theta=$ angle between $R$ and $H$, and $\Psi=$ dihedral angle.]

$$
\mathbf{A}_{\mathrm{CG}}=\pi \mathbf{X}^{2}=\pi\left(\mathbf{R}^{2}-\mathbf{H}^{2}\right)=3 \pi \mathbf{R}^{2}\left(1-\cos ^{2} \theta\right)
$$

$$
\mathbf{A}_{\mathbf{P}}=\mathbf{A}_{\text {esfera }}-6 \mathbf{A}_{\text {calota }}=2 \pi \mathbf{R}^{2}[2-6(1-\cos \theta)]
$$

$$
\mathbf{V}_{\mathbf{G}}=\mathbf{V}_{\text {esfera }}-6 \mathbf{V}_{\text {calota }}=\frac{\pi \mathbf{R}^{3}}{3}\left[4-6(1-\cos \theta)^{2}(2+\cos \theta)\right]
$$

$$
\mathbf{V}_{\mathbf{P}}=\mathbf{V}_{\text {cubo }}-\mathbf{V}_{\mathbf{G}}=8(\mathbf{H})^{3}-\mathbf{V}_{\mathbf{G}}=8(\mathbf{R} \cos \theta)^{3}-\mathbf{V}_{\mathbf{G}}
$$

Todas as grandezas podem ser calculadas conhecendo-se as variáveis $\mathrm{R}$ e $\mathrm{H}$ (lembrando-se que $\cos \theta=\mathrm{H} / \mathrm{R}$ ).

A partir do cálculo das grandezas para a célula unitária, ou seja, para um grão, é possível fazer o mesmo para um grama de grãos considerando-se que o número de grão por grama do material, $\mathrm{N}$, pode ser obtido através da razão do volume de um grão pelo volume por grama do material $\left(\mathrm{V}_{\text {grama }}\right.$ ou inverso da densidade teórica $\left.=1 / \rho_{\mathrm{t}}\right)$ segundo a relação:

$$
\mathbf{N}=\frac{\mathbf{V}_{\text {grama }}}{\mathbf{V}_{\mathbf{G}}}=\frac{1}{\mathbf{V}_{\mathbf{G}} \rho_{\mathbf{t}}}
$$

$\mathrm{A}$ área do contorno de grão $\left(\overline{\mathrm{A}_{\mathrm{CG}}}\right)$, a área dos poros $\left(\overline{A_{P}}\right)$, e o volume dos poros $\left(\overline{V_{P}}\right)$ para um grama de amostra são obtidos multiplicando-se o número de grãos, $\mathrm{N}$ - equação $(\mathrm{E})$, pelas relações das equações $\mathrm{A}, \mathrm{B}$ e $\mathrm{D}$, respectivamente.

A densidade aparente do material $(\rho)$ pode ser calculada conhecendo-se pela relação:

$$
\rho=\frac{1}{\overline{\mathbf{V}_{\mathrm{P}}}+\frac{1}{\rho}}
$$

Através da equação (F) é possível também verificar a 
mudança da densidade conhecendo-se a variação de $\mathrm{R}$ e $\mathrm{H}$.

Uma vez estabelecidas as relações geométricas que possibilitam o cálculo de $\overline{\mathbf{A}_{\mathrm{CG}}}, \overline{\mathbf{A}_{\mathrm{P}}}$, e $\rho$ através do conhecimento de $\mathrm{R}$ e $\mathrm{H}$ é possível realizar a simulação de algumas condições específicas da mudança de $\mathrm{H}$ em função da mudança do raio R. Três casos serão simulados:

1) $\mathrm{O}$ primeiro considerando o número de grãos, $\mathrm{N}$, constante. É preciso lembrar que o princípio de conservação de massa deve ser respeitado em todos os casos. No primeiro caso o volume do grão é constante;

2) $\mathrm{O}$ segundo caso em que $\mathrm{N}$ diminui com o tempo, ou seja, crescimento de grãos. Nesta simulação a razão foi considerada constante em função do tempo e $0<\mathrm{H} / \mathrm{R}<1$ ( $\cos \theta=$ constante)

3) $\mathrm{O}$ terceiro caso considerou-se que $\mathrm{N}$ diminui com o tempo, ou seja ocorre também crescimento de grãos, contudo a razão $\mathrm{H} / \mathrm{R}$ diminui com o tempo mas $\mathrm{H}$ aumenta proporcional e linearmente com o aumento de $\mathrm{R}$ ainda com $0<\mathrm{H} / \mathrm{R}<1$ ( $\cos \theta=$ variável com o tempo constante).

As três condições propostas têm o objetivo de simular os casos observados durante a sinterização de partículas, ou seja: densificação sem crescimento de partículas, coalescência de grãos sem densificação e densificação com crescimento de grãos, respectivamente.

Primeira simulação: número de grãos constante e volume de um grão constante.

O primeiro estudo foi realizado considerando que o número de grãos é constante e tem por conseqüência que o volume de um grão é também constante. A variação da razão $H / R$ permite que sejam determinadas as variação de, $\overline{\mathbf{A}_{\mathrm{CG}}}$ e $\overline{\mathbf{A}_{\mathrm{P}}} \rho$ (para um material com densidade igual a $1 \mathrm{~g} . \mathrm{cm}^{-3}$ e raio inicial igual a $1 \mu \mathrm{m}$ ) e que estão representados na Fig. 2 (todos os valores de área são dados em $\mathrm{m}^{2} / \mathrm{g}$ ).

Verifica-se que ocorre a diminuição da $\overline{\mathbf{A}_{\mathrm{P}}}, \mathrm{o}$ aumento

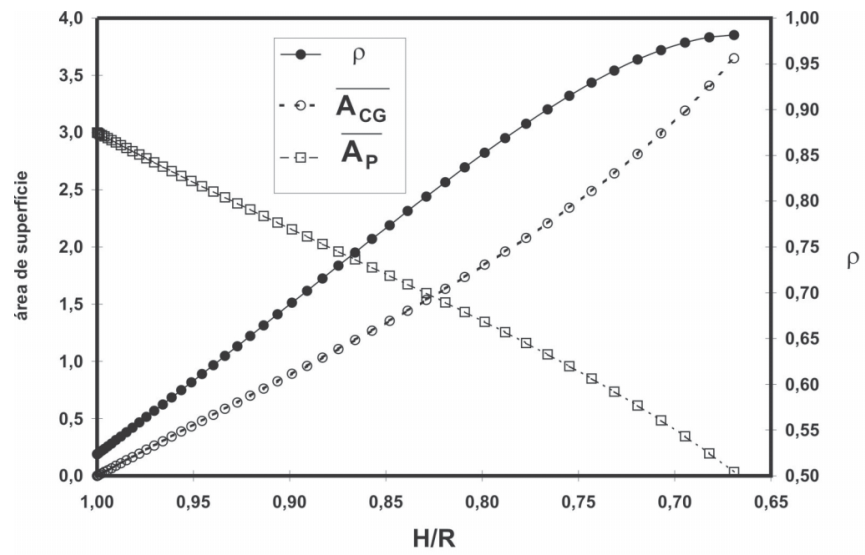

Figura 2: Variação de, $\overline{\mathbf{A}_{\mathrm{CG}}}, \overline{\mathbf{A}_{\mathrm{P}}}$ e $\rho$ com H/R, considerando-se o volume e o número de grãos constantes.

[Figure 2: $\overline{\mathbf{A}_{\mathrm{CG}}}, \overline{\mathbf{A}_{\mathbf{P}}}$ and $\rho$ variation with the $H / R$ ratio (volume of the grains $=$ constant).] simultâneo de $\overline{\mathbf{A}_{\mathrm{CG}}}$, bem como da densificação do material, como previsto pelos modelos de densificação sem crescimento de grãos. O modelo geométrico adotado permite que o cálculo seja realizado para ângulos pouco superiores a $45^{\circ}$, pois a partir destes valores existe interação entre mais de dois grãos para a formação do mesmo contorno de grão e o modelo matemático não é mais válido.

A consideração de que o número de grãos é constante tem uma conseqüência ligada à conservação de massa do sistema. A aproximação do centro das esferas com a diminuição de $\mathrm{H}$ e o volume dos grãos constante leva ao aumento de $\mathrm{R}$ devido à conservação de massa do sistema. Desta forma, mantendo o volume do grão constante é possível calcular a variação de R em função de H (Fig. 3).

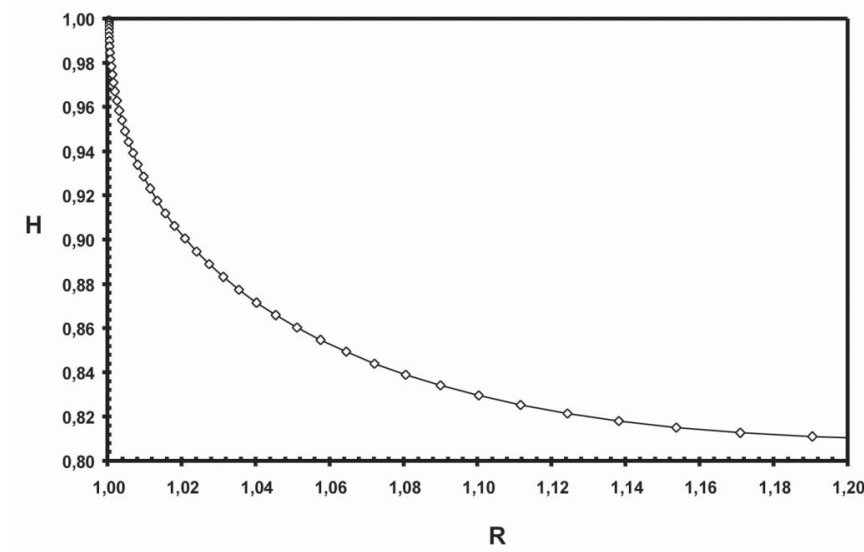

Figura 3: Variação de $\mathrm{H}$ com $\mathrm{R}$ durante a densificação sem crescimento de grãos (volume dos grãos = constante).

[Figure 3: $H$ versus $R$ during simulation of densification without grain growth (grains volume = constant).]

O raio da parte esférica pode crescer até cerca de $20 \%$ para uma diminuição da mesma proporção de H. Vale evidenciar que ocorre uma variação inicial bastante rápida para $\mathrm{H}$ enquanto $\mathrm{R}$ permanece praticamente constante como previsto para modelos de sinterização baseados em formação de pescoço entre as partículas. Contudo, para estados adiantados de densificação, $\mathrm{R}$ deve crescer numa proporção maior que $\mathrm{H}$ e a aproximação feita nos modelos de estágio inicial de sinterização deve ser válida somente para o início da formação de pescoço $(H / R<0,95)$. A diferença entre a energia da superfície e da interface deve ser elevada nessa condição, já que deve existir a minimização da energia livre do sistema segundo a equação $(G)$ e a variação das duas superfícies é a razão entre as variações das duas interfaces é muito próxima de um.

$$
\mathbf{d G}=\gamma_{\mathrm{P}} \mathbf{d A _ { P }}+\gamma_{\mathrm{CG}} \mathbf{d A _ { C G }}
$$

Como a variação da área do contorno de grão é positiva, para que o sistema seja termodinamicamente favorável é preciso que a energia da superfície (ou poro) seja maior que a do contorno de grão. Este é a consideração energética proposta nos modelos tradicionais. 
Segunda simulação: crescimento de grãos com $H / R=$ constante.

A segunda simulação foi realizada considerando-se que a taxa $H / R$ permanece constante enquanto o número de grãos diminui, ou seja, no caso em que ocorre coalescência de partículas. A aproximação do centro dos grãos ocorre na mesma proporção que o raio das partículas aumenta. Esta consideração leva em conta que o mesmo fenômeno que produz o aumento do tamanho de partículas leva também ao aumento da distância do centro das mesmas e numa proporção constante e igual. Desta forma, à medida que os grãos crescem, a distância entre o centro dos grãos cresce em uma mesma proporção, levando a uma condição de crescimento de grãos com crescimento simultâneo dos poros. Pode-se então esperar que a simulação do modelo nestas condições leve a diminuição proporcional de $\overline{\mathbf{A}_{\mathrm{P}}}$ e $\overline{\mathbf{A}_{\mathrm{CG}}}$ sem que haja aproximação do centro das partículas (densidade constante). $\mathrm{O}$ crescimento de grãos em função do tempo pode ser simulado através de uma equação típica do tipo "Ostwald ripening" para crescimento de partículas [3]:

$$
\mathbf{R}^{\mathbf{n}}-\mathbf{R}_{0}^{\mathbf{n}}=\mathbf{B} \mathbf{t}
$$

onde B é uma constante que depende da temperatura, do coeficiente de difusão e da tensão superficial e té o tempo. n é determinado pelo tipo de mecanismo envolvido no crescimento de grãos, que para Ostwald ripening é igual a 3.

O resultado da simulação para, $\overline{\mathbf{A}_{\mathrm{CG}}}, \overline{\mathbf{A}_{\mathrm{P}}}$ e $\boldsymbol{\rho}$ considerando: $\mathrm{R}_{0}=1, \mathrm{n}=3, \mathrm{~B}=10, \rho_{\mathrm{t}}=1$ e $\mathrm{H} / \mathrm{R}=0,985$ (que corresponde a $\theta=10)$ é apresentado na Fig. 4.

A simulação mostra que a densidade permanece constante quando a distância entre os grãos, $\mathrm{H}$, cresce na mesma proporção que R. Entretanto, ocorre uma diminuição de $\overline{\mathbf{A}_{\mathrm{CG}}}$ e $\overline{\mathbf{A}_{\mathrm{P}}} \mathrm{em}$ função do tempo. Uma visão mais detalhada é dada na Fig. 5.

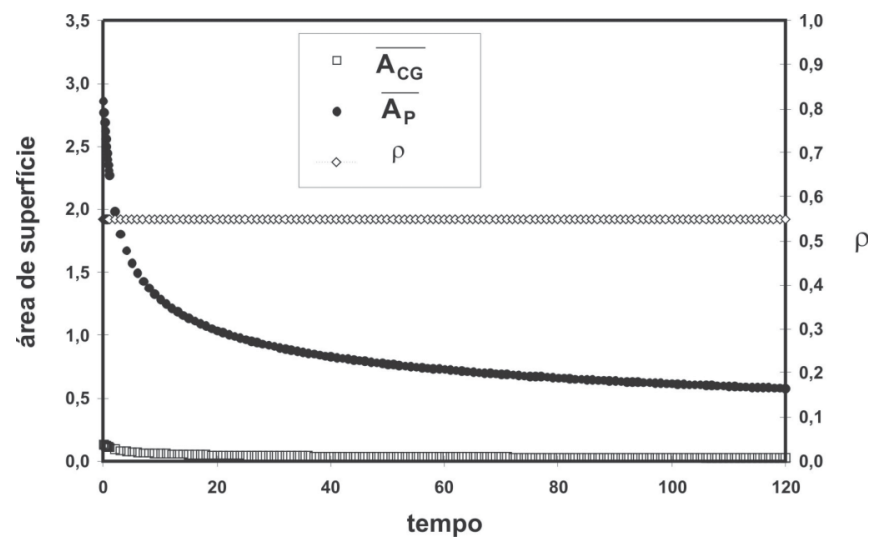

Figura 4: Variação de, $\overline{\mathbf{A}_{\mathrm{CG}}}$ e $\overline{\mathbf{A}_{\mathrm{P}}}$ e $\boldsymbol{\rho}$ durante o crescimento dos grãos mantendo a relação de $\mathrm{H} / \mathrm{R}$ constante $(\operatorname{com} \mathrm{H} / \mathrm{R}=0,984 \mathrm{ou}$ $\theta=10)$.

[Figure 4: $\overline{\mathbf{A}_{\mathrm{CG}}}$ e $\overline{\mathbf{A}_{\mathrm{P}}}$, and $\boldsymbol{\rho}$ variation during simulation of grain growth with the ratio $H / R=$ constant.]

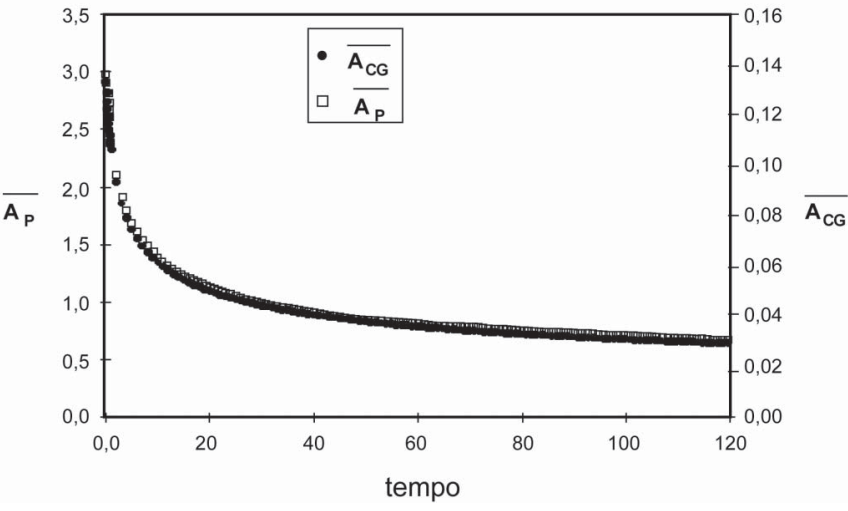

Figura 5: Variação de $\overline{\mathbf{A}_{\mathrm{CG}}}$ e $\overline{\mathbf{A}_{\mathrm{P}}}$ com o crescimento de grãos em função do tempo para a relação de $\mathrm{H} / \mathrm{R}=0,984$.

[Figure 5: Kinetics of $\overline{\mathbf{A}_{\mathrm{CG}}}$ and $\overline{\mathbf{A}_{\mathbf{P}}}$ with grain growth for $H / R=0.984$.

Tanto $\overline{\mathbf{A}_{\mathrm{CG}}}$ como $\overline{\mathbf{A}_{\mathrm{P}}}$ diminuem com o crescimento de partículas. Esta diminuição simultânea das áreas esta de acordo com o princípio termodinâmico da sinterização apresentado pela equação $(\mathrm{G})$. Esta situação deve ser mais favorável energeticamente que aquela apresentada anteriormente, já que a diminuição da energia livre será maior.

Apesar de $\overline{\mathbf{A}_{\mathrm{CG}}}$ e $\overline{\mathbf{A}_{\mathrm{P}}}$ serem de ordens de grandeza diferentes, as suas variações são iguais. Na verdade na condição de crescimento de grãos com coalescência para a

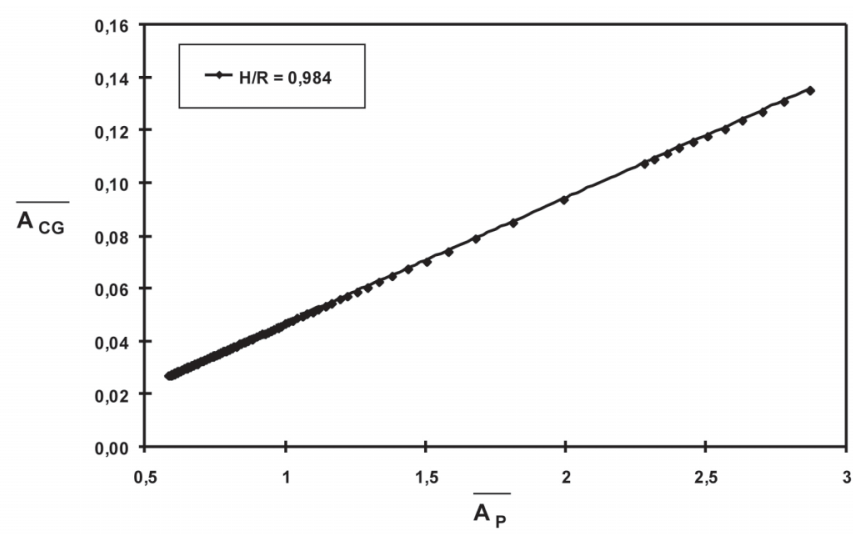

Figura 6: Variação de $\overline{\mathbf{A}_{\mathrm{CG}}}$ com $\overline{\mathbf{A}_{\mathrm{P}}}$ durante o crescimento de grãos para $\mathrm{H} / \mathrm{R}=0,984$.

[Figure 6: $\overline{\mathbf{A}_{\mathrm{CG}}}$ versus $\overline{\mathbf{A}_{\mathrm{P}}}$ during grain growth for $H / R=0.984$.]

razão $\mathrm{H} / \mathrm{R}=$ constante, a variação de $\overline{\mathbf{A}_{\mathrm{CG}}}$ com $\overline{\mathbf{A}_{\mathrm{P}}}$ é linear como pode ser visto na Fig. 6.

A relação de $\overline{\mathbf{A}_{C G}}$ e $\overline{\mathbf{A}_{\mathrm{P}}}$ é linear passando pela origem, ou seja, para que $\overline{A_{C G}}$ seja igual a zero, $\overline{A_{P}}$ deve ser zero. A relação observada entre $\overline{A_{C G}}$ e $\overline{A_{P}}$ é semelhante àquela observada na sinterização do $\mathrm{SnO}_{2}$ sem aditivos [4]. A sinterização desse sistema é conhecida por apresentar 
crescimento de grãos e poros sem densificação [5].

Realizando a simulação para um outro valor constante da razão $H / R(H / R=0,866$ ou $\theta=30)$ são obtidos resultados numéricos diferentes, mas comportamentos idênticos, ou seja, densidade constante e variação linear entre $\overline{\mathbf{A}_{\mathrm{CG}}}$ e $\overline{\mathbf{A}_{\mathrm{P}}}$ como pode ser visto na Fig. 7.
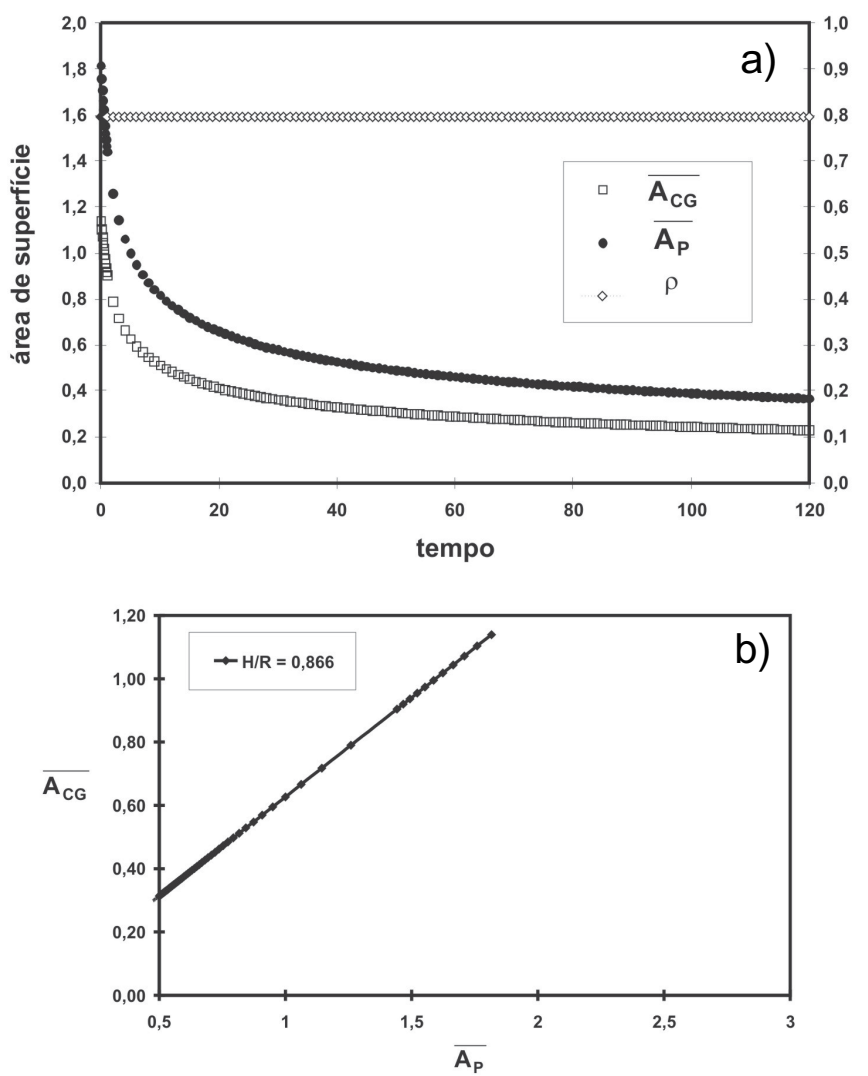

Figura 7: Simulação para a razão $H / R=0,866\left(\theta=30^{\circ}\right)$ mostrando que a densidade permanece constante e $\overline{\mathbf{A}_{\mathrm{CG}}}$ e $\overline{\mathbf{A}_{\mathrm{P}}}$ diminuem (a) e a variação linear entre $\overline{\mathbf{A}_{\mathrm{CG}}} \mathrm{e} \overline{\mathbf{A}_{\mathrm{P}}}$ (b).

[Figure 7: Simulation of the, $\overline{\mathbf{A}_{\mathrm{CG}}}, \overline{\mathbf{A}_{\mathbf{P}}}$ and $\boldsymbol{\rho}$ with $H / R=0.866$ $\left(\theta=30^{\circ}\right)$ (a) and linear relationship between $\overline{\mathbf{A}_{\mathrm{CG}}}$ and $\overline{\mathbf{A}_{\mathbf{P}}}(b)$.]

Outra relação interessante pode ser verificada entre a variação da área total, $\overline{\mathbf{A}_{T}}$, que é igual a $\overline{\mathbf{A}_{T}}=\overline{\mathbf{A}_{\mathrm{CG}}}+\overline{\mathbf{A}_{\mathrm{P}}}$, e o inverso do raio dos grãos. Pode ser facilmente demonstrado que, para um determinado número de grãos esféricos e para um grama de material, a área total por grama de material é dada pela relação [1]:

$$
\overline{\mathbf{A}_{\mathbf{T}}}=\frac{3}{\rho \mathbf{R}}
$$

onde $\overline{A_{T}}$ é a área específica em $\mathrm{m}^{2} \cdot \mathrm{g}^{-1}, \rho$ é a densidade em g.cm ${ }^{-3}$ e R em $\mu \mathrm{m}$. Foi verificado para a simulação numérica que a relação representada pela equação (I) permanece válida durante o crescimento dos grãos na presença de contornos nas
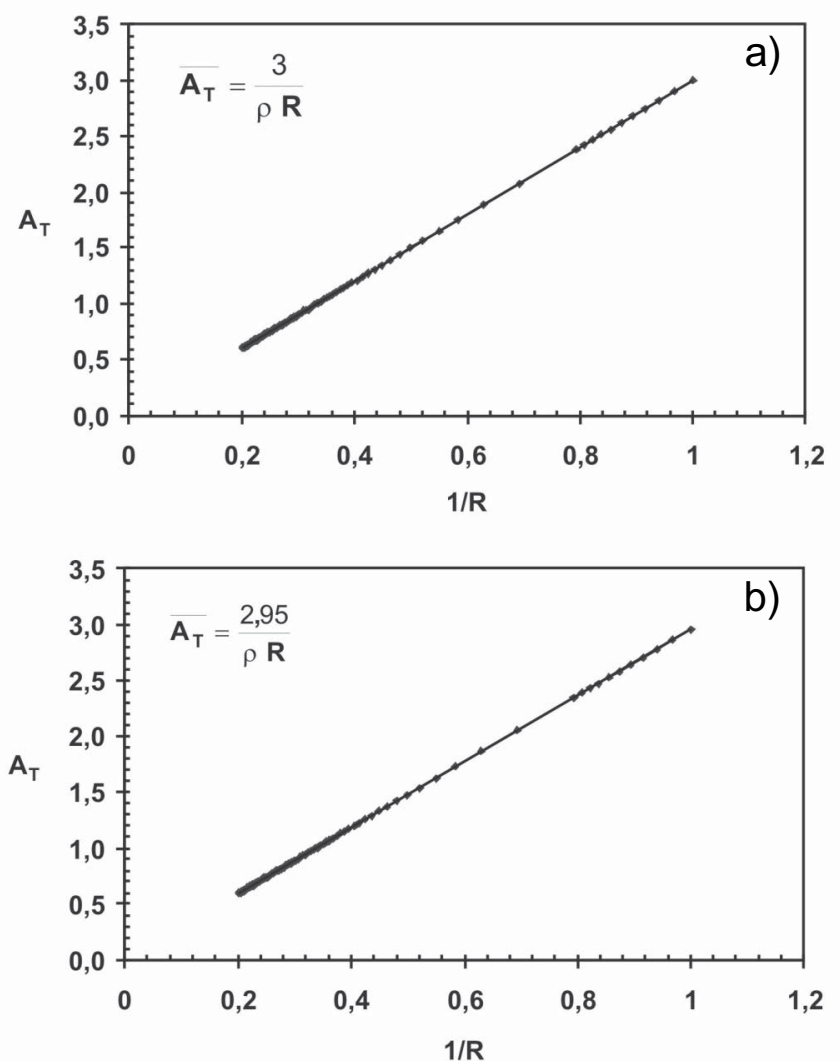

Figura 8: Variação de $\overline{\mathbf{A}_{\mathrm{T}}}$ com $1 / \mathrm{R}$ durante a simulação de crescimento de grãos com $\mathrm{dH} / \mathrm{dR}=1$ e (a) $\mathrm{H} / \mathrm{R}=0,984$ e (b) $\mathrm{H} / \mathrm{R}=0,866$.

[Figure 8: $\overline{\mathbf{A}_{\mathrm{T}}}$ versus $1 / R$ for grain growth simulation with $d H / d R=1$ (a) $H / R=0.984$ and (b) $H / R=0.866$.]

duas simulações, como pode ser visto na Fig. 8.

A variação de $\overline{\mathbf{A}_{C G}}$ e $\overline{\mathbf{A}_{\mathrm{P}}}$ com $1 / \mathrm{R}$ também pode ser verificada e nestas condições é linear como pode ser visto na Fig. 9 (para $\mathrm{H} / \mathrm{R}=0,984)$.

Em resumo pode-se concluir que para a simulação do sistema com $\mathrm{H} / \mathrm{R}=$ constante e $0<\mathrm{H} / \mathrm{R}<1$, temos:

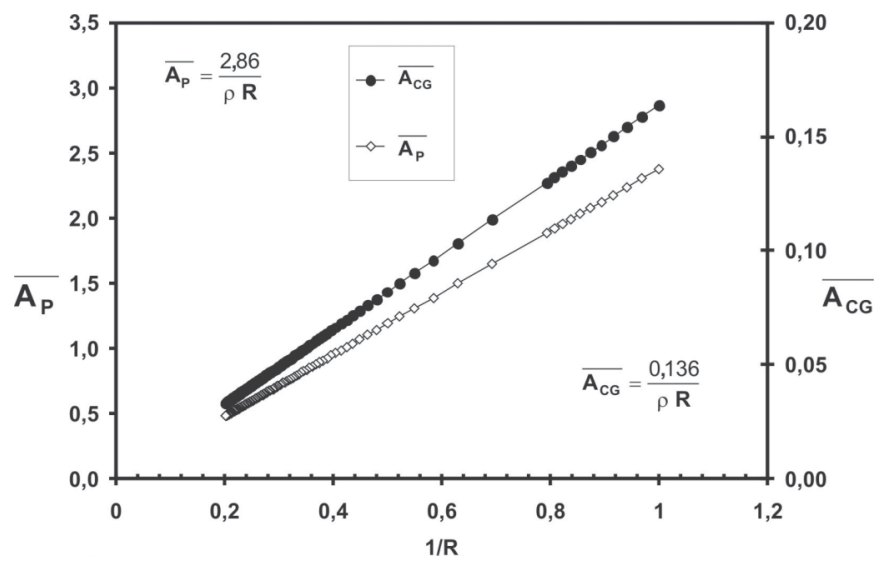

Figura 9: Variação de $\overline{\mathbf{A}_{C G}}$ e $\overline{\mathbf{A}_{\mathbf{P}}} \operatorname{com} 1 / \mathrm{R}($ para $H / R=0,984)$.

[Figure 9: Simulation of $\overline{\mathbf{A}_{\mathrm{CG}}}$ e $\overline{\mathbf{A}_{\mathrm{P}}}$ with $1 / R(H / R=0.984)$ ] 
1. Durante o crescimento dos grãos a densidade permanece constante.

2. Apesar do volume total de poros permanecer constante, o tamanho dos poros cresce. Ou seja, ocorre crescimento de grãos e poros simultaneamente sem que haja densificação.

3.Existe uma relação linear em $\overline{\mathbf{A}_{\mathrm{P}}}$ e $\overline{\mathbf{A}_{\mathrm{CG}}}$ durante a coalescência das partículas semelhante a sistemas reais.

4. A relação geométrica entre a área total dos grãos e o raio das partículas $\overline{\mathbf{A}_{\mathrm{T}}}=\frac{3}{\rho \mathbf{R}}$ continua válida durante o crescimento das partículas sem densificação.

5. Existe uma relação linear entre $\overline{A_{P}}$ e $1 / R$ e também entre $\overline{\mathbf{A}_{\mathrm{CG}}}$ e $1 / \mathrm{R}$.

6. A diminuição de energia livre neste caso deve ser superior àquela com densificação sem crescimento de grãos, já que a superfície e a interface são eliminadas simultaneamente.

Terceira simulação: crescimento de grãos com $H / R$ não constante, mas $H$ variando linearmente com $R(0<H / R<1$ e $\cos \theta=$ variável com o tempo)

A terceira simulação considera que a distância entre os grãos representado por $\mathrm{H}$ cresce durante o crescimento de grãos, mas com uma taxa menor que R. Isto significa que durante o crescimento dos grãos deve ocorrer a aproximação dos centros dos grãos e, por conseqüência, a densificação. Nesta condição a densificação fica condicionada ao crescimento de grãos pela taxa $\mathrm{dH} / \mathrm{dR}$, ou seja, quanto menor for $\mathrm{dH} / \mathrm{dR}$ maior será a aproximação do centro dos grãos durante o aumento de $\mathrm{R}$.

A integração da equação $\mathrm{dH} / \mathrm{dR}=\mathrm{A}$ (com A constante $\mathrm{e}$ $0<\mathrm{A}<1$ ) resulta em:

$$
\begin{aligned}
& \int_{H_{0}}^{H} d H=A \int_{R_{0}}^{R} d R \\
& H-H_{0}=A\left(R-R_{0}\right)
\end{aligned}
$$

Como $\mathrm{H}_{0}$ é igual a $\mathrm{R}_{0}$ então:

$$
\mathbf{H}=\mathbf{A}\left(\mathbf{R}-\mathbf{R}_{0}\right)+\mathbf{R}_{0}
$$

Desta maneira, $\mathrm{H}$ cresce de forma linear com o crescimento de R. Conhecendo como R varia com o tempo é então possível o cálculo de $\mathrm{H}$ e também de $\overline{\mathbf{A}_{\mathrm{P}}}$ e $\overline{\mathbf{A}_{\mathrm{CG}}}$ e $\rho$ em função do tempo.

O crescimento de partículas foi simulado pela equação $(H)$, com $\mathrm{R}_{0}=1, \mathrm{n}=3, \mathrm{~B}=10, \rho_{\mathrm{T}}=1$ para diferentes valores de dH/dR: 0,$985 ; 0,940 ; 0,866 ; 0,766$ e 0,707 .

A variação da densidade para diferentes valores $\mathrm{de} \mathrm{dH} / \mathrm{dR}$ é apresentada na Fig. 10.

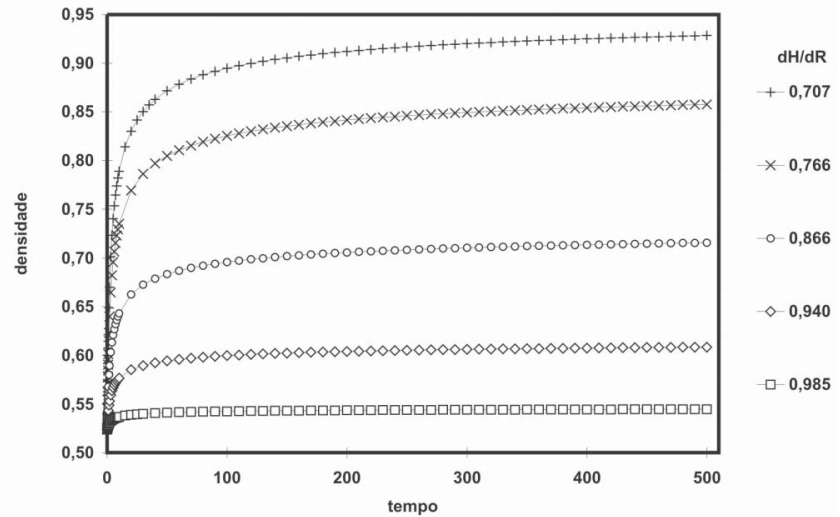

Figura 10: Simulação da densidade em função do tempo para diferentes valores de $\mathrm{dH} / \mathrm{dR}$.

[Figure 10: Simulation of the densification kinetics for different dH/dR.]

A simulação mostra que quanto menor $\mathrm{dH} / \mathrm{dR}$ maior a densidade final obtida no mesmo tempo. A variação da densidade em função do tempo apresenta grande semelhança com curvas experimentais de cinética de densificação em condições isotérmicas, que é caracterizada por uma forte densificação nos momentos iniciais de sinterização, tendendo a uma densidade de equilíbrio, mesmo que seja menor que a densidade teórica. A simulação da sinterização com densificação e crescimento de grãos foi obtida sem a divisão do processo em diferentes etapas e de forma satisfatória. As áreas do contorno de grãos e dos poros foram calculadas e são apresentados a seguir.

A variação de $\overline{\mathbf{A}_{C G}}$ com o tempo para diferentes valores de dH/dR são apresentados na Fig. 11.

$\overline{\mathbf{A}_{\mathrm{CG}}}$ apresenta um forte crescimento nos momentos iniciais e corresponde à etapa onde há uma densificação intensa, para em seguida diminuir com o tempo. É interessante observar que nestas condições ocorre a diminuição da área total do contorno de grãos a partir de um determinado instante e que a área de contorno formada é maior quanto menor $\mathrm{dH} / \mathrm{dR}$. Este comportamento foi observado durante a sinterização de alumina por Shaw e Brook [6]. A diminuição da área durante

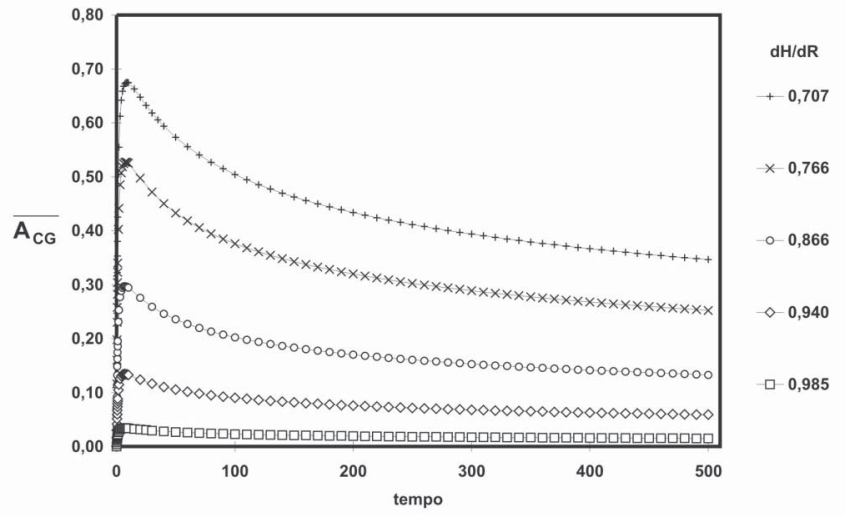

Figura 11: Simulação de $\overline{\mathbf{A}_{\mathrm{CG}}}$ com o tempo para diferentes valores de $\mathrm{dH} / \mathrm{dR}$.

[Figure 11: Simulation of the $\overline{\mathbf{A}_{\mathrm{CG}}}$ kinetics for different dH/dR.] 


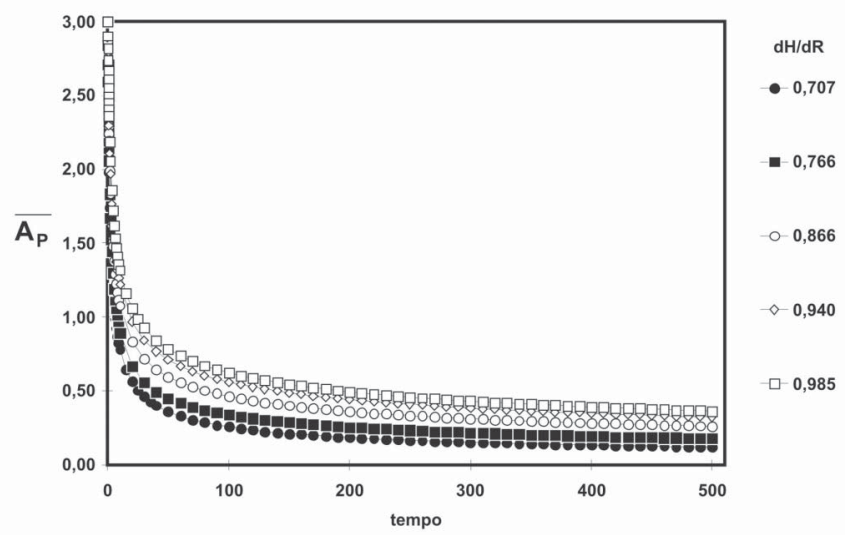

Figura 12: Variação de $\overline{\mathbf{A}_{\mathrm{P}}}$ com o tempo para diferentes valores de $\mathrm{dH} / \mathrm{dR}$.

[Figure 12: Simulation of the $\overline{\mathbf{A}_{\mathrm{P}}}$ kinetics for different dH/dR.]

o processo de densificação é esperada, já que o sistema deve ser conduzido a uma diminuição da energia livre total do sistema - equação $(\mathrm{G})$. A diminuição simultânea da área de contorno leva a uma menor área final de contorno de grãos e a uma menor energia total do sistema.

A variação de $\overline{\mathbf{A}_{p}}$ em função do tempo para diferentes valores de dH/dR é mostrada na Fig. 12.

A simulação de $\overline{\mathbf{A}_{\mathrm{P}}}$ em função do tempo de sinterização mostra um decréscimo mais acentuado da área de poro para $\mathrm{dH} / \mathrm{dR}$ menores, ou seja, onde a densificação é mais efetiva. A diminuição da área de poros era esperada, levando em conta o argumento da diminuição da energia livre, como no caso anterior. Desta forma, a coalescência de grãos deve ocorrer sempre, já que na sinterização com ou sem densificação, o crescimento de partículas leva a uma minimização da energia livre do sistema.

A relação linear entre a área total (área do contorno + área dos poros) em função do inverso do raio do grão se mantém como observado para a simulação de coalescência de grãos (Fig. 13). Vários autores consideram que o modelo

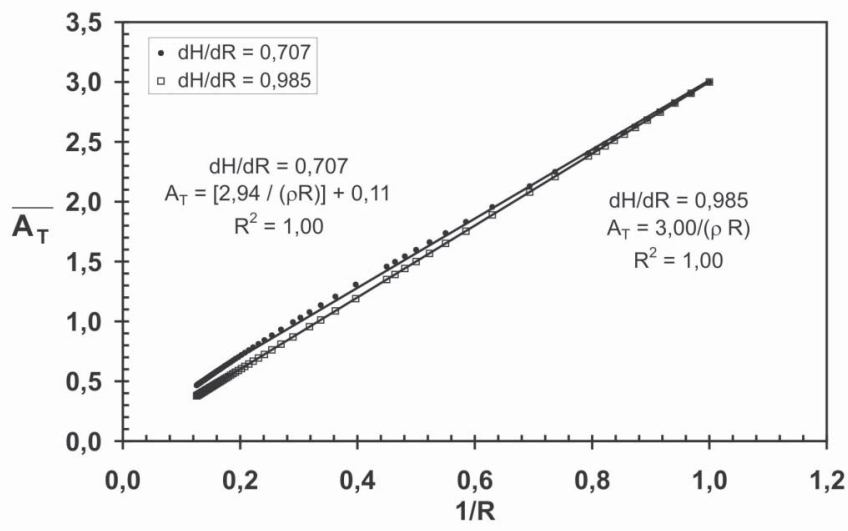

Figura 13: Variação de $\overline{\mathbf{A}_{\mathrm{T}}}$ com $1 / \mathrm{R}$ durante a simulação de crescimento de grãos com dH/dR=1 e (a) $\mathrm{H} / \mathrm{R}=0,984$ e (b) $\mathrm{H} / \mathrm{R}=0,866$.

[Figure 13: $\overline{\mathbf{A}_{\mathrm{T}}}$ versus $1 / R$ during simulation of grain growth with $d H / d R=1$ and (a) $H / R=0.984$ and (b) $H / R=0.866$.] representado pela equação (I) só é válido para esferas. Em função dos resultados obtidos da simulação é possível considerar que a área dos poros varia linearmente com o inverso do tamanho dos grãos.

As conclusões parciais alcançadas na simulação com $H / R$ variável com $\mathrm{H}$ variando linearmente com $\mathrm{R}$ foram:

1. As simulações apresentam uma continuidade e não é necessário dividir o fenômeno em diferentes etapas.

2. As curvas de densidade em função do tempo são semelhantes àquelas obtidas experimentalmente.

3. O aumento da área de contorno de grãos ocorre nos momentos iniciais de sinterização. O crescimento dos grãos faz com que haja uma diminuição da área do contorno de grãos ao mesmo tempo em que ocorre a diminuição da área dos poros. Este tipo de comportamento é mais adequado ao princípio de diminuição de energia livre do sistema.

4. A relação do inverso do tamanho de grãos e da área total pode ser considerada linear durante todo o processo.

\section{Considerações termodinâmicas}

A variação de $\mathrm{H} / \mathrm{R}$ com o tempo foi calculada para diferentes razões de dH/dR como no caso anterior e é apresentada na Fig. 14. Em função da razão escolhida entre $\mathrm{dH} / \mathrm{dR}$ a razão $\mathrm{H} / \mathrm{R}$ tende a um valor constante diferente, assim como o ângulo $\theta$, já que $\cos \theta=\mathrm{H} / \mathrm{R}$. Como $\theta=\Psi / 2$ onde $\Psi$ é o ângulo diedro (Fig. 1) e este está diretamente relacionado ao equilíbrio das energias da interface e da superfície segundo a equação $(\mathrm{M})[3,7]$ :

$$
\cos (\psi / 2)=\frac{\gamma_{\mathrm{CG}}}{2 \gamma}
$$

a igualdade pode ser escrita:

$$
\cos (\psi / 2)=\frac{\gamma_{\mathrm{CG}}}{2 \gamma_{\mathrm{P}}}=\cos \theta=\frac{\mathrm{H}}{\mathrm{R}}
$$

Existe uma relação direta entre a razão $H / R$ e a razão entre as energias. H/R torna-se praticamente constante em função do tempo segundo as simulações realizadas (Fig. 14) o que significa que o ângulo diedro tende a um ângulo de equilíbrio. Este resultado vai ao encontro das considerações mais recentes sobre sinterização [8-10], que assumem que existe uma evolução da microestrutura sempre na direção de se obter um ângulo de equilíbrio onde o sistema apresenta uma menor energia.

Os papeis da energia do poro e do contorno de grão na densificação podem ser discutidos e parecem estar em acordo com os resultados apresentados por Castro e Gouvêa [9]. No caso em que a razão H/R é constante, implica em que $\gamma_{\mathrm{CG}} / 2 \gamma_{\mathrm{P}}$ é constante. Mesmo que haja variação dos valores das energias, a sua razão permanece constante durante o processo de sinterização e, neste caso, existe uma ausência da eliminação dos poros. Como a razão entre as energias é constante, durante o processo de sinterização as duas superfícies são eliminadas simultaneamente na mesma taxa e não ocorre aproximação 


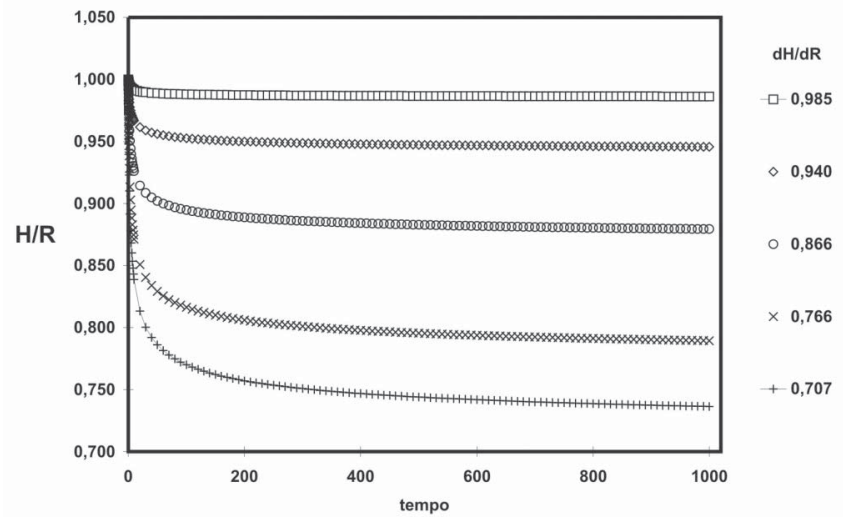

Figura 14: Variação de H/R com o tempo para diferentes valores de $\mathrm{dH} / \mathrm{dR}$.

[Figure 14: Kinetics of $H / R$ for different $d H / d R$ values.]

relativa do centro das partículas.

No caso da simulação de $\mathrm{H} / \mathrm{R}$ variando com o tempo e variação linear de $\mathrm{H}$ com $\mathrm{R}$, mas $\mathrm{h} / \mathrm{R}$ tende a um uma razão de equilíbrio que corresponde a uma razão $\gamma_{C G} / 2 \gamma_{\mathrm{P}}$ de equilíbrio, ou seja, a um ângulo diedro de equilíbrio, como proposto por vários trabalhos recentes $[7,8]$. A densificação ocorre, já que neste caso a energia do contorno de grão é inferior à do poro apresentando assim maior estabilidade e será em um primeiro momento formada e em um segundo momento, eliminada durante o crescimento dos grãos.

O conceito de modificação das energias de poro e de contorno de grãos foi utilizado com sucesso durante a sinterização do $\mathrm{SnO}_{2}$ contendo $5 \%$ em mol de $\mathrm{Fe}_{2} \mathrm{O}_{3}$ onde foi possível a densificação das amostras em 10 segundos de isoterma quando sinterizadas por "fast firing" [10].

\section{CONCLUSÕES}

As simulações apresentadas demonstram que a relação entre $H / R$ pode ser determinante para a sinterização de um compacto de partículas. Quando H/R é constante durante o crescimento de grãos foi calculada uma ausência de densificação, mas eliminação de área do contorno de grão e de poros de forma simultânea, como observado durante a sinterização do $\mathrm{SnO}_{2}$ sem aditivos. Já quando $\mathrm{H} / \mathrm{R}$ varia com o tempo com $\mathrm{dH} / \mathrm{dR}=$ constante (com valores entre 0 e 1 ) e $\mathrm{H}$ varia linearmente com $\mathrm{R}$, é observada a aproximação do centro das esferas e assim eliminação de poros. As curvas de densidade em função do tempo parecem simular de forma satisfatória o comportamento da sinterização de materiais cerâmicos. Uma forte densificação inicial é observada nos primeiros momentos da sinterização onde ocorre um aumento da área de contorno de grão. Contudo, com o crescimento das esferas, é observada uma eliminação da superfície do contorno de grão. Os dois casos parecem melhor satisfazer as condições da minimização da energia livre do sistema quando comparada com a situação em que há densificação sem crescimento de grãos. A cinética de crescimento de grão foi mantida constante para as diferentes razões $\mathrm{dH} / \mathrm{dR}$ e desta forma a densificação não parece ser afetada pela cinética de crescimento de partículas, mas unicamente pela razão $\mathrm{dH} / \mathrm{dR}$.

A relação evidente entre $\mathrm{H} / \mathrm{R}$ e a razão das energias $\gamma_{\mathrm{CG}} / 2 \gamma_{\mathrm{P}}$ demonstra que a as energias da superfície e do contorno de grão pode ser determinante para a densificação ou não de um sistema de partículas. A consideração de que os aditivos podem modificar de forma importante a relação de energias e não somente os coeficientes de difusão do sistema pode levar a uma forma diferente de se abordar o problema da densificação de materiais cerâmicos e possibilitar inovações tecnológicas.

Trabalhos estão sendo realizados na tentativa de demonstrar a adequação dos resultados experimentais com as simulações e apresentam resultados promissores na interpretação da sinterização de vários óxidos cerâmicos.

\section{REFERÊNCIAS}

[1] M. N. Rahaman, Ceramic processing and sintering, Marcel Dekker, New York (1995) p. 333.

[2] G. J. Pereira, R. H. R. Castro, P. Hidalgo, D. Gouvêa, Surface segregation of additives on $\mathrm{SnO}_{2}$ based powders and their relationship with macroscopic properties, Appl. Surf. Sci. 195 (2002) 277.

[3] Yet-Ming Chiang, D. P. Birnie, W. D. Kingery, Physical Ceramics - Principles for ceramic science and engeneering, John Wiley \& Sons, New York (1997) p. 388.

[4] D. Gouvêa, Efeito da segregação do manganês na morfologia dos pós e na sinterização do $\mathrm{SnO}_{2}$, Tese de Doutorado, Universidade Federal de S. Carlos (1995).

[5] J. A. Varela, E. Longo, N. Bareli, A. S. Tanaka, W.A Mariano, Sinterização do óxido de estanho em várias atmosferas, Cerâmica 31 (1985) 241.

[6] N. J. Shaw, R. J. Brook, Structure and coarsening during the sintering of alumina, J. Am. Ceram. Soc. 69, 2 (1986) 107. [7] F. Wakai, F. Aldinger, Equilibrium configuration of particles in sintering under constraint, Acta Mater. 51 (2003) 641.

[8] J. L. Shi, Thermodynamics and densification kinetics in solidstate sintering of ceramics, J. Mater. Res. 14, 4 (1999) 1398.

[9] J. L. Shi, Relation between coarsening and densification and mass transport path in solid-state sintering of ceramics: model analysis, Mater. Res. 14, 4 (1999) 1378.

[10] F. F. Lange, B. J. Kellett, Thermodynamics of densification: II, grain growth in porous compacts and relation to densification, J. Am. Ceram. Soc. 72, 5 (1989) 735.

[11] D. Gouvêa, R. H. R. Castro, Sintering: The role of interface energies, App. Surf. Sci. 271, 1-4 (2003) 194.

[12] G. J. Pereira, D. Gouvêa, Densificação rápida de cerâmicas de $\mathrm{SnO}_{2}$, Cerâmica 49, 310 (2003) 116.

(Rec. 05/03/03, Rev. 05/03/04, Ac. 16/04/04) 